\title{
Effective Parameters on Nanostructured Solar Cell
}

\section{Efficiency}

\author{
Bassam Ghalib Rasheed \\ Applied sciences department, University of Technology, Baghdad 18310, Iraq
}

Received: December 16, 2012 / Accepted: January 08, 2013 / Published: April 25, 2013.

\begin{abstract}
Performance of the nanostructured solar cell prepared by photoelectrochemical etching has been studied. Various affecting parameters on the nanostructured solar cell have been examined. It is found that the cell efficiency was increased from $12 \%$ to $18.5 \%$ when optimum current density of $15 \mathrm{~mA} / \mathrm{cm}^{2}$ was used. While increasing the illumination light intensity at the solar cell top surface with blue light leads to enhance the cell efficiency. The surface morphology investigation reveals formation of nanostructured surface with pore size distribution affected by the preparation conditions. The scanning probe microscopy implies that uniform nanostructured surface of narrow pore size distribution with mean value of $25 \mathrm{~nm}$ could be obtained when optimum preparation condition are employed.
\end{abstract}

Key words: Nanostructured solar cell, silicon nanostructures, photoelectrochemical etching, porous silicon.

\section{Introduction}

An explosion of interest in silicon nanoparticles was set off in 1990 with Canham's discovery of the photoluminescence (PL) properties of porous silicon which attributed to the quantum confinement (QC) of carriers [1]. Silicon nanoparticles/nanocrystals play a particular role among various materials nanostructures. This may be attributed to many reasons like the low cost, good accessible in addition to low toxicity and fully compatible. The interest comes mainly from the large role that silicon could play in the emerging technology of optoelectronics, as it has already been successful in microelectronics [2]. The most important applications of porous silicon (PS) device consisting of silicon nanostructures in optoelectronics, specifically solar cells. A silicon solar cell is considered as a common device for conversion of sunlight to an electrical current. These kinds of solar cells contribute to about $94 \%$ of the word market and

Corresponding author: Bassam Ghalib Rasheed, assistant professor, research fields: nanotechnology, nanostructured materials and materials processing. E-mail: dr_bassamghalib@yahoo.com. most are made from crystalline silicon. An early application of porous $\mathrm{Si}$ in photovoltaics was reported more than 15 years ago. The work was addressed for the minimization of optical losses in mono and polycrystalline silicon based solar cells. The anodic dissolution of the ordinary solar cells was carried out and the porous layer was penetrated the $p-n$ junction through the active top layer of the solar cell, which is extremely very thin (less than $1 \mu \mathrm{m}$ ) [3].

The low conversion efficiencies, which do not exceed $20 \%$, as well as the high manufacturing cost of ordinary solar cells (due to the high antireflection layer coating costs) are considered the greatest limitations of conventional silicon solar cells. These limitations correspond to the reversibility of the photon absorption process and the single gap nature of the silicon. However, such limitations could be overcome by synthesis of multi-band gap materials of large surface area. Nanostructured solar cells based on ordinary $p-n$ junction of controllable surface features could enhance the conversion efficiency and reduce the maintenance requirements, as well. Improvement of such limitations could make it possible to 
manufacture inexpensive solar cells [4].

Many research activities have been devoted on solar cells to enhance the conversion efficiency. The beginning was started when a research group [5] formed a porous silicon (PS) structure consisting of nanostructures on silicon solar cells by both electrochemical and photoelectrochemical etching methods. They created a porous layer of $500 \mathrm{~nm}$ thickness on a polycrystalline wafer. This work followed by investigating the optical detection of the solar cell. It is found that the reflection of the porous layer was reduced from $90 \%$ to $70 \%$ in the visible region [6].

Porous silicon could also be formed by stain etching in aqueous $\mathrm{HF} / \mathrm{HNO}_{3}$ solution. The optical properties of the prepared films were investigated by reflectance spectroscopy [7]. This porous layer could be used as an antireflection coating in simplified processing for solar cells. The efficiency of such solar cell achieved of about $17 \%$ [8].

Moreover, the nanostructured surface could also be produced by a vapor etching method. The porous silicon layer in addition to its good antireflection action acts as passivating agent for surface and substrate regions of the cell [9]. Furthermore, the structural stability of the porous layer planned to be used in solar cell technology. It was found that when the fresh porous layer is subjected to free air oxidation, the surface reflection is reduced [10].

The chemical etching technique was used to texture the surface of the polycrystaliine silicon solar cell [11]. Moreover, silicon nanowire based on solar cell could also be fabricated to improve the conversion efficiency of the solar cell. Thin amorphous silicon layers were deposited on nanowire array to form the p-n junction [12]. A one-dimensional photonic crystal can be used to study the nanostructured surface and determine its features [13]. The surface morphology and reflectivity of the porous layers both are remarkably changed and it $\mathrm{t}$ is found that both are easily modulated by controlling the preparation conditions [14].

The aim of this work is to synthesis efficient nanostructured solar cells by photoelectrochemical etching process. The performance of the nanostructured solar cell is expected to be higher than that of ordinary solar cells. Therefore, this nanostructured solar cell would reduce the number of the required cells compared with the normal solar cells due to its higher efficiency. Moreover, this process offers a low cost with good efficiency which reduces the maintenance costs.

\section{Experiments}

Conventional solar cell of $400 \mu \mathrm{m}$ thicknesses was used as raw materials in this work. This solar cell has a conversion efficiency of $12 \%$. The main challenge in this work is to adopt the etching process on the solar cell surface without affecting the underlying junction characteristics. Therefore, etching cell made from Teflon was designed and constructed as shown in Fig. 1 to synthesize nanostructured layer at the top of the solar cell. Etching only one side of the solar cell has been considered in this design, while the backside of the solar cell is completely separated from the etching acid, thereby, the metallization of the backside which is coated by aluminum will be protected. Photoelectrochemical etching technique was employed

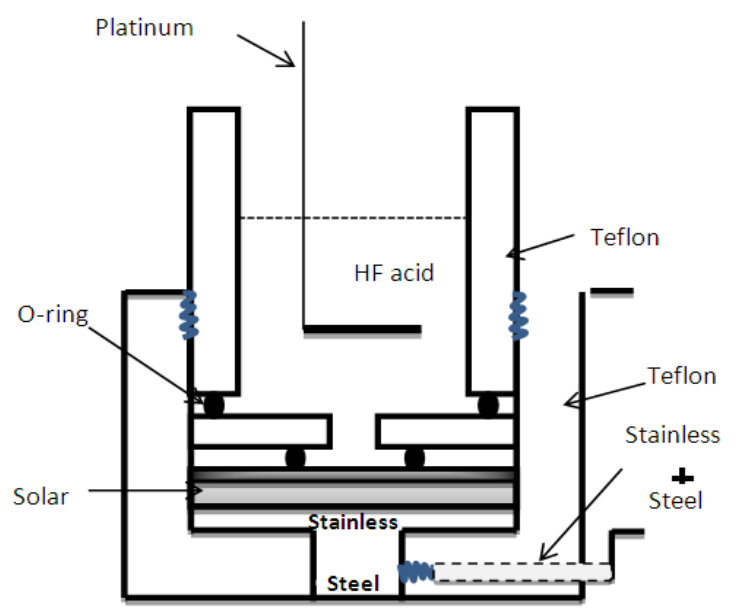

Fig. 1 Schematic diagram of the etching cell used in this work. 
to synthesize a nanostructured surface at the top of the solar cell (n-type side) using a blue light emerged from a $500 \mathrm{~W}$ halogen lamp. In order to achieve a homogenous and uniform porous structure layer, an ohmic contact is required on the entire backside surface of the cell. The area of the solar surface that could be etched in this design is $4.5 \mathrm{~cm}^{2}$.

The performance of the solar cell could be determined by measuring the conversion efficiency $(\eta)$, fill factor $(F . F)$ and the open circuit voltage $\left(V_{O C}\right)$ as well as the short circuit current $\left(I_{S C}\right)$.

The fill factor is calculated using the relation [15]:

$$
F . F=\frac{P_{\max }}{V_{o c} I_{s c}}
$$

where, $P_{\max }=I_{m} \times V_{m}$, While the cell efficiency $\eta$ is given by:

$$
\eta=P_{\max } / P_{A M}
$$

$$
\eta=\frac{F \cdot F V_{O C} I_{S C}}{P_{A M} 1.5}
$$

Initial investigation involves the surface morphology of the ordinary solar cells using a high resolution optical microscope were conducted to study the surface topography before the texturing process. Then, the optical microscope images were analyzed using a computer image processing software. High resolution optical microscope (Olympus $\mathrm{BH}_{4}$ ) was used to study the microstructure of the surface and SPM (Angstrom 2000) was also used to study the nanostructures morphology of the surface. The optoelectronic properties of the nanostructured solar cells were measured under the standard illumination AM1.5 $\left(100 \mathrm{~W} / \mathrm{cm}^{2}\right)$.

\section{Results and Discussion}

Both optoelectronic and morphological properties were examined in this study to obtain higher performance of the nanostructured solar cell.

\subsection{Optoelectronic Properties}

The optoelectronic properties of the solar cell have been investigated in this study since those properties determine how much the incident sunlight could be converted to photocurrent.
The photoelectrochemical etching technique is considered as most significant technology to produce, modify and control of nanostructured layer at both n-type and p-type silicon respectively. The formation of macroporous layer in silicon with pore diameter larger than $50 \mathrm{~nm}$ by electrochemical dissolution in hydrofluoric acid solution could be exposed to the light simultaneously to modify and control the porous layer features and reduce the pore diameters to less than $10 \mathrm{~nm}$. This technique is a self-regulating process determined by the current density at the end of the pore.

In this work, effects of three effective parameters on the optoelectronic characteristics of the solar cell were examined: etching current density, HF acid solution and the illumination light intensity. To study one parameter, others should be kept fixed.

The etching current density is expected to be one of the most significant parameter which rules the formation of the nanostructured porous layer. It is found that the etching current density was very effective on the solar cell properties as shown in Fig. 2. The solar cell etched in $10 \% \mathrm{HF}$ acid concentration diluted in water and ethanol 1:1:2, respectively, and illumination light intensity of 2,000 lux. The solar cell efficiency $\eta$ reach its optimum value at certain current density $15 \mathrm{~mA} / \mathrm{cm}^{2}$ as given in Table 1 . The etching

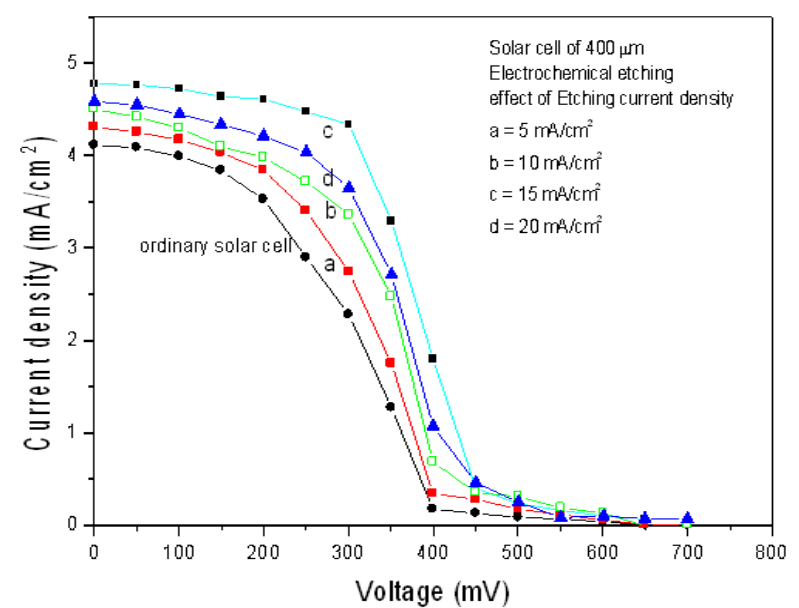

Fig. 2 The optoelectronic properties of the nanostructured solar cell prepared by photoelectrochemical etching under different current density. 


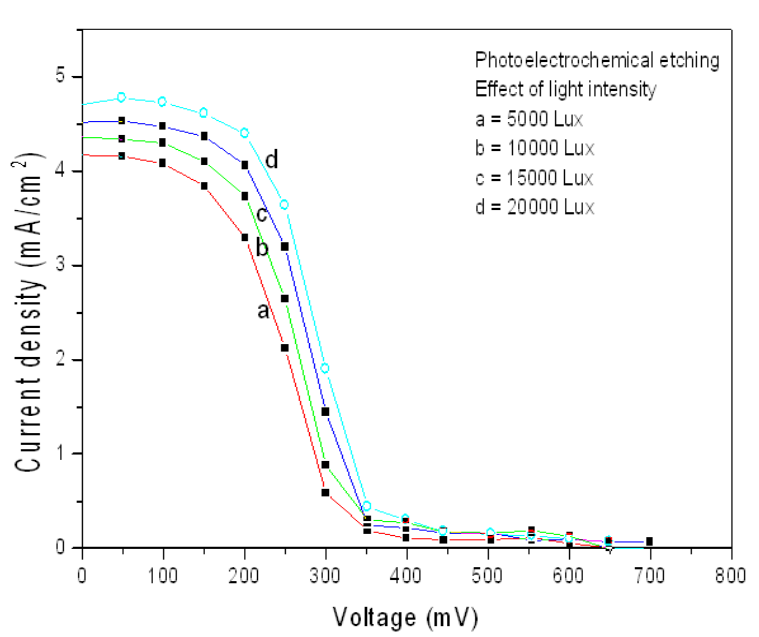

Fig. 3 The optoelectronic properties of the nanostructured solar cell prepared by photoelectrochemical etching under illumination of different light intensity.

current density is responsible on holes accumulation at the surface. Farther increase of the current density greater than the optimum value leads to consume holes in much higher speed compared with holes accumulation speed and derives the chemical reaction toward the electro-polishing process then decreases the porous layer thickness.

The HF acid solution consideration was found very effective parameter and played a significant role on the etching rate (etching speed) of the photoelectrochemical etching. The optimum value of the current density of $15 \mathrm{~mA} / \mathrm{cm}^{2}$ and illumination light intensity of 2,000 lux were employed. Table 2 shows that increasing the HF concentration from $10 \%$ to $30 \%$ leads to increase the etching rate from 0.15 $\mu / \mathrm{min}$ to $0.5 \mu \mathrm{m} / \mathrm{min}$ and this is attributed to the increment of the effective fluorine ions which attack the silicon top surface and consequently detach the silicon atoms from the surface. Since this chemical reaction is very slow, it is preferable to use low HF concentration in order to achieve cell of higher efficiency [16].

Effect of the illumination light intensity has also been examined. Fig. 3 explains effect of the illumination light intensity on the optoelectronic solar cell at $10 \mathrm{~mA} / \mathrm{cm}^{2}$ current density and $10 \% \mathrm{HF}$ concentration. It is found that illumination with higher light intensities improve the cell efficiency. The cell efficiency increases for $12 \%$ for the ordinary solar cell to $15.4 \%$ when the nanostructured solar cell is subjected to light of 2,000 lux, as given in Table 3 . This could be attributed to effect of the additional holes which generated by the light absorption. These holes added to the supplied holes by the external power supply. Ultimately, the overall accumulated holes are consumed by the chemical reaction. We pointed out that the light generated holes are generated slowly and inconsistence with the chemical reaction speed which is also slow. While if the holes supplied faster, the result may take a negative trend. Table 1 gives the solar cell characteristics prepared under different illumination light intensity.

The solar cell could convert the incident light to photocurrent when it is subjected to reverse biasing. This biasing helps to diffuse the generated charge carriers to the electrodes before the recombination

Table 1 Characteristics of the electrochemically etched solar cell of $\mathbf{4 0 0} \boldsymbol{\mu m}$ under different current densities.

\begin{tabular}{cll}
\hline Current density $\left(\mathrm{mA} / \mathrm{cm}^{2}\right)$ & Efficiency & Fill Factor \\
\hline Ordinary solar cell of $400 \mu \mathrm{m}$ & 12 & 0.7 \\
5 & 14 & 0.72 \\
10 & 14.8 & 0.74 \\
15 & 18.5 & 0.78 \\
20 & 13.8 & 0.72 \\
\hline
\end{tabular}

Table 2 The etching rate with different HF acid concentrations for photoelectrochemical etching.

\begin{tabular}{ll}
\hline HF concentration $(\%)$ & Etching rate $(\mu \mathrm{m} / \mathrm{min})$ \\
\hline 10 & 0.15 \\
20 & 0.25 \\
30 & 0.50 \\
40 & 0.80 \\
\hline
\end{tabular}

Table 3 Characteristics of the nanostructured solar cell of $400 \mu \mathrm{m}$ thickness prepared by photoelectrochemical etching with different light intensity.

\begin{tabular}{llc}
\hline Efficiency & Fill Factor & Light Intensity (Lux) \\
\hline 12 & 0.7 & Ordinary solar cell \\
13.4 & 0.72 & 5,000 \\
14.8 & 0.74 & 10,000 \\
17.5 & 0.76 & 15,000 \\
18.5 & 0.78 & 20,000 \\
\hline
\end{tabular}


process takes place. Since different preparation conditions could affect the nanostructured layer formation, optimization of these conditions should be obtained.

The J-V curves of the solar cells are measured under illumination of the standard AM1.5 (100 $\mathrm{mW} / \mathrm{cm}^{2}$ ). Fig. 4 represents the J-V properties of the ordinary solar cells and the nanostructured solar cell of optimum efficiency (current density of $15 \mathrm{~mA} / \mathrm{cm}^{2}$, HF concentration of $10 \%$ and light intensity of 20,000 lux). The efficiency $\eta$ of the nanostructured solar cell has improved from $12 \%$ to $18.5 \%$ due to the light absorption enhancement.

\subsection{The Surface Morphology}

To characterize the solar cell surface features, surface morphology investigations are required. This study involves the microstructure and nanostructure features and their analysis. The microstructural properties were examined using a high resolution optical microscope.

The porous layer which is synthesized by electrochemical etching could be highly controlled with the presence of light. When the top solar cell surface exposed to the light during the electrochemical etching, the photogenerated holes reconstruct the surface and modify the texturing process. It is necessary to point out that using white light illumination leads to produce inhomogeneous structure at the surface due to the effect of various penetration depths or different existent wavelengths. Long wavelengths have deeper penetration depth would damage the junction since the n-type layer is abundant at the top surface of the solar cell which is very thin (some times less than $1 \mu \mathrm{m}$ ). Therefore, blue light emerged from halogen lamp has been used to obtain a small enough penetration depth and avoid disturbing the junction. To explain how much the surface morphology is affected by this process. Fig. 5 shows the surface morphology of the solar cell top surface before and after texturing the top solar surface

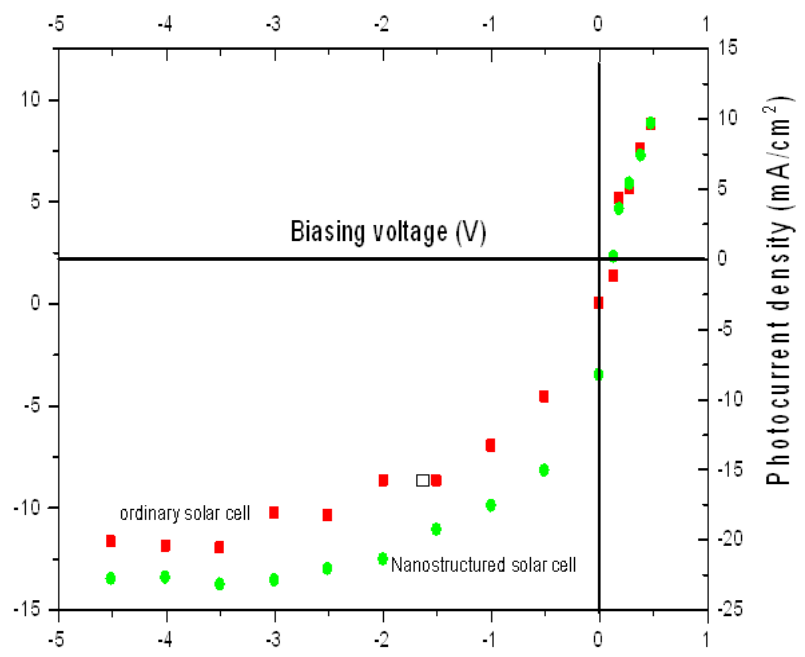

Fig. 4 The J-V characteristics of ordinary and nanostructured solar cells.

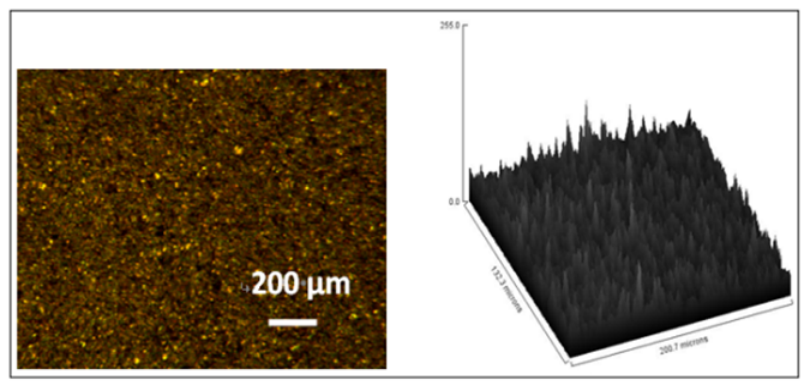

(a)

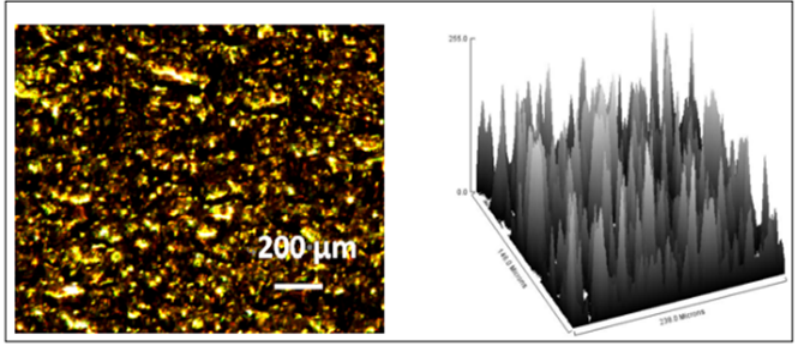

(b)

Fig. 5 Micrograph of high resolution optical microscope and the surface plot for the ordinary solar cell top surface (a) before and (b) after surface texturing, respectively.

by photo electrochemical etching with light assistance. The surface plot reveals remarkable differences in the surface morphology before and after the texturing process.

It is found that the surface topography of the nanostructured solar cell surface shows various structures when different preparation conditions are employed. The surface morphology of the porous layer has extremely rich details with respect of range 


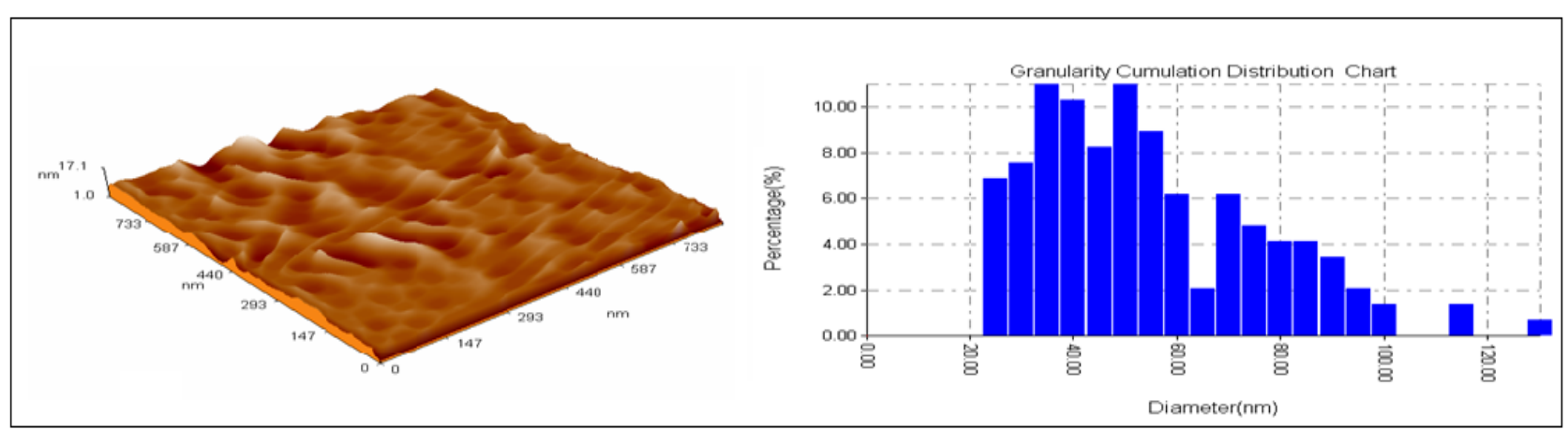

(a)

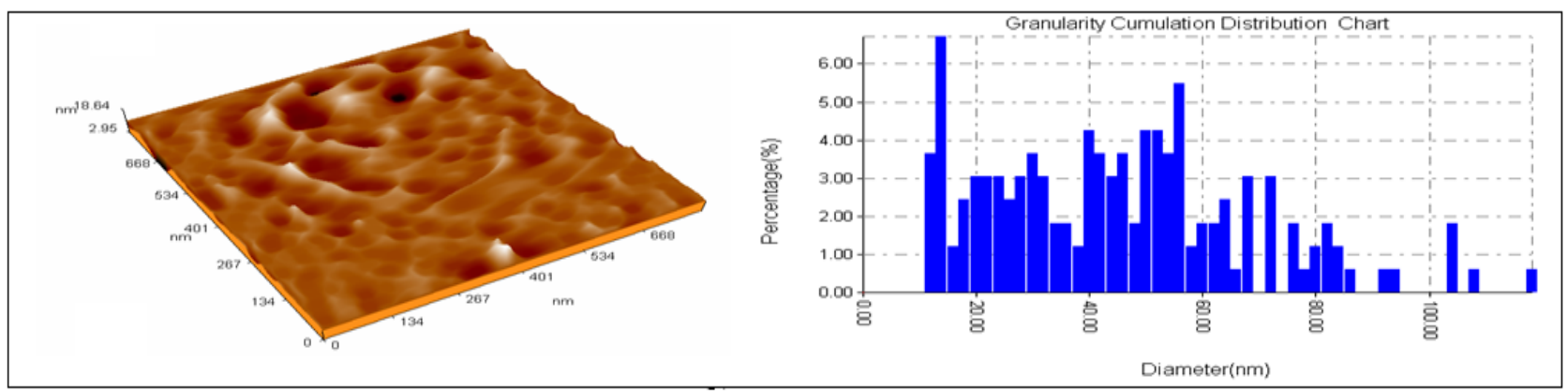

(b)

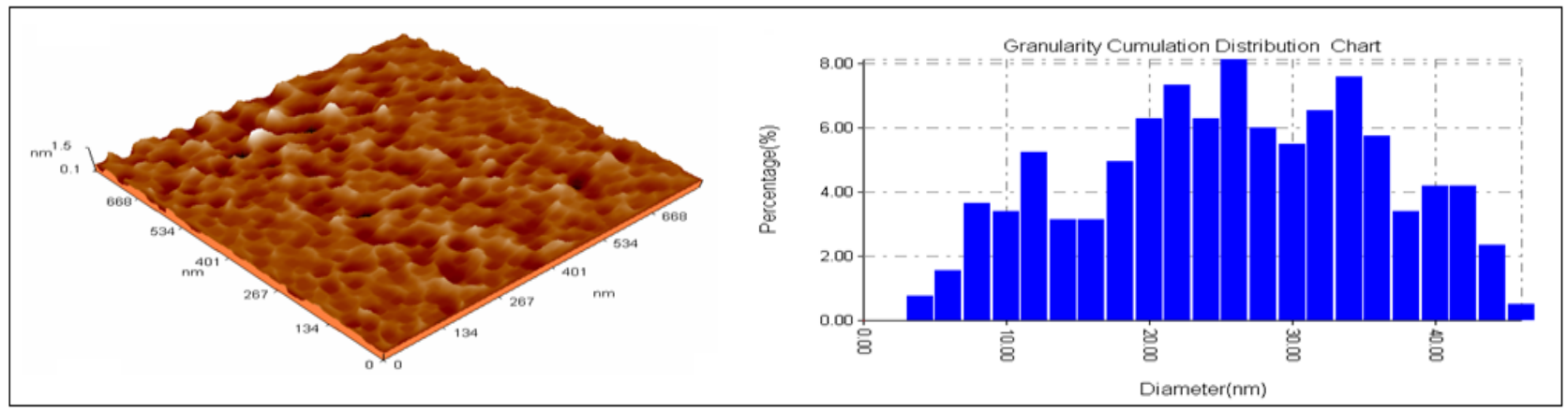

(c)

Fig. 6 The SPM of the top surface of nanostructured solar cell prepared by photoelectrochemical etching process and their pore distribution.

of variation in the pore size, shape and their distribution. The scanning probe microscopy investigation reveal a formation of nanostructured surface of different features and pore distribution. Fig. 6 illustrates the surface morphology of the nanostructured solar cell in the left column with their pore distribution and percentage abundance in the right column. When a relatively high current density of $25 \mathrm{~mA} / \mathrm{cm}^{2}$ is used, low surface roughness was obtained as shown in Fig. 6a. The pore diameter distribution shows uniform pore distribution but with pore mean diameter of $(50 \mathrm{~nm})$. While for high
HF concentration of $30 \%$, the surface morphology reflects a less roughness of (35 nm) as shown in Fig. $6 \mathrm{~b}$ and non-uniform pore distribution of pore mean diameter of (40 nm). Better surface morphology (Fig. $6 \mathrm{c}$ ) of optimum roughness of $10 \mathrm{~nm}$ was obtained when current density of $15 \mathrm{~mA} / \mathrm{cm}^{2}$ with $10 \% \mathrm{HF}$ concentration and 20,000 lux light illumination intensity. This structure has a uniform and narrow pore distribution with mean diameter of $25 \mathrm{~nm}$.

\section{Conclusions}

The solar cell performance could be enhanced by 
texturing solar cell top surface by photoelectrochemical etching process. Holes consumption should be adjusted with holes accumulation to control the chemical reaction between $\mathrm{HF}$ acid and silicon solar cell. The etching current density has an optimum value which produces nanostructured solar cell of high efficiency. The surface morphology is affected by many parameters and could be controlled to obtain a uniform nanostructured surface of narrow pore size distribution using optimum preparation conditions.

\section{Acknowledgment}

Acknowledgment and appreciation for CRDF \& Arab Science and Technology foundation for their financial support.

\section{References}

[1] L. Canham, Silicon quantum wire array fabrication by electrochemical and chemical dissolution of wafers, Appl. Phys. Lett. 57 (1990) 1064.

[2] C. Poole, J. Owens, Introduction to Nanotechnology, John Wiley \& Sons inc. (2005) 20-25.

[3] Y. Zhao, W Sang, The influence of microstructure on optical properties of porous silicon, Solid State Electronics 51 (2007) 680.

[4] H. Kawamoto, K. Okuwada, Surface oxidation of luminescence porous silicon, Sci. \& Tech. Quarterly Rev. 24 (2007) 39.

[5] P. Menna, G. di Franci, Porous silicon in solar cells: A review and a description of its application in an $\mathrm{AR}$ coating, Solar Energy Materials \& Solar Cells 37 (1995) 13.

[6] E. Kolesar, V. Bright, Optical reflectance reduction of silicon surface coated with antireflection thin film, Thin Solid Films 290-291 (1996) 23.

[7] L. Schirone, F. Califano, Chemically coated porous silicon as antireflection coating for high efficiency solar cells, Thin Solid Films 297 (1997) 296.

[8] R. Bilyalov, L. Stalmans, Use of porous silicon antireflection coating in multi crystalline silicon solar cell processing, IEEE Trans. Electron Devices 46 (10) (1999) 2035.

[9] W. Dimassi, M. Saadoun, Porous silicon-based passivation and gettering in poly crystalline silicon solar cell, Nuc. Meth. In Phys. Res. B 186 (2002) 441.

[10] S. Aouida, M. Saadoun, Structural and luminescence properties of vapor etched porous silicon, Phys. Status Solidi, 2(2005) 3409.

[11] P. Panek, M. Lipi, 'Texturization of multi crystalline silicon by wet chemical etching for silicon solar cell, J. Mat. Science 40 (2005) 1459.

[12] L. Taskalakos, J. Balch, Silicon nanowire solar cells, Appl. Phys. Lett. 91 (2007) 233117.

[13] N. Fuke and A. Fukui, Electron transport in back contact dye-sensitized solar cell, J. Appl. Phys. 104 (2008) 64307.

[14] Z. Nansheng, M. Zhongquan, Lower reflectivity and higher minority carrier life time of hand-tailored porous silicon, J. of Semiconductors 30 (2009) 72004.

[15] J. Torre, G. Bremond, Using silicon nanostructures for the improvement of silicon solar cell efficiency, Thin Solid Films 511-512, (2006) 161.

[16] B. Rasheed, A. Al-hamdani, Y. Dawood, Properties of nanostructured solar cell prepared at different etching time, J. Mat. Sci. \& Eng. 4 (2010) 3. 